\title{
3D Dendrite Reconstruction and Spine Identification
}

\author{
Wengang Zhou ${ }^{1}$, Houqiang $\mathrm{Li}^{1}$, and Xiaobo Zhou ${ }^{2}$ \\ ${ }^{1}$ Department of EEIS, University of Science and Technology of China, Hefei, P.R. China \\ ${ }^{2}$ Center of Biotechnology and Informatics, The Methodist Hospital \\ Research Institute \& Weill Medical College of Cornell University, \\ Houston, TX 77030 \\ zhwg@mail.ustc.edu.cn, lihq@ustc.edu.cn, XZhou@tmhs.org
}

\begin{abstract}
In neuron-biology, 3D neuron dendrite reconstruction followed by spine identification is indispensable for the study of neuronal functions and biophysical properties. In this paper, we propose an automatic dendrite reconstruction method to with a surface representation of the neuron on the basis of a novel level set approach. Our novel level set approach can effectively tackle the problem of segmentation under blurring and intensity in-homogeneity. Then spines are detected based on dendrite medial axis and a label-based thinning strategy is proposed to accurately extract the dendrite skeleton for spine identification. Experimental results reveal that our method works well.
\end{abstract}

Keywords: Dendrite, spine, reconstruction, level set, segmentation, skeleton.

\section{Introduction}

Dendritic spines, or spines for short, are small protrusion on the dendrite, a tree-like structure of neuron cell. Spines play an important role in many neurological cognitive functions such as memory, experience-based learning, and mental retardation, et al [1]. The structure of neuronal dendrites and their spines underlie the connectivity of neural networks and may be important predicators of their function. To investigate the neuronal functions and biophysical properties, accurate three-dimensional reconstruction of neuron dendrite cells is indispensable.

Many existing methods of neuron reconstruction are semi-automatic and may require manual intervention to identify salient structures and deal with ambiguities in each confocal microscopy data-set independently [2]. Some reconstruction methods represent neuronal structures as a tree of connected cylinders [3]. Strong assumptions about the objects of interest are made. Other methods employ curve-skeleton methods to represent dendritic structures with some graph model [4]. The skeleton is obtained either through segmentation followed by thinning or by filtering to extract center points. However, the skeletonisation algorithm employed may be sensitive to small changes on the object surface and the resulting skeleton may not represent the neuron topology accurately.

The analysis of dendritic structure and morphology is mostly carried out manually which is extremely time-consuming and irreproducible. To reduce manual labor and 
improve the accuracy and reproducibility of the analysis, some spine detection and quantification methods of varying degrees of automation have been suggested. Some researchers adopt the medial axis to identify spines in 2D as protrusions relative to dendritic skeleton [5], with limited accuracy. Some use model based methods to detect spines [6]. These methods don't work well in detection of short spines or spine with short neck. Others use 3D medial axis-based strategies to extract a skeleton and identify spurs as potential spines [7]. Medial axis based methods are sensitive to noise and heuristics have to be applied to delete false positives.

In this paper, we present an automatic method for dendrite reconstruction followed by spine identification. We choose to reconstruct the dendrite with the neuron surface. Neuron dendrites are segmented through a new level set approach which can effectively tackle blurring and intensity in-homogeneity. With the new segmentation method, the general morphology and details of segmented dendrites are satisfactorily preserved. As an intrinsic property of active contours with level set evolution, the surface of segmented dendrite is smooth, thus it provides a favorable property for reconstruction with surface. Then spines are detected and through a label-based 3D thinning strategy the skeleton is extracted which can be used to accurately identify spines. Our method is automatic and reconstruction with accuracy and reliability is achieved.

This paper is organized as follows. Section 2 gives the image acquisition and processing methods. Section 3 describes the neuron dendrite reconstruction and spine identification analysis. Section 4 discusses our result and validation. Finally, we conclude the paper in section 5 .

\section{Image Acquisition and Processing}

\subsection{Data-Set Acquisition}

3D neuron dendrite images were acquired by the digitization of neuronal cultures using a two photon laser scanning microscopy with a $40 *$ objective and 0.8 NA. Due to the nature of the image acquisition process and the photo-bleaching effects, the acquired neuron dendrite images are characterized by some features. First, because of the microscopy's point spread function, images are blurred with out-of-focus. Second, photon shot noise is popular in image domain. Third, some unrelated structures are present. Fourth, many spine heads are often observed as detached form the main dendrite structure. These features make it difficult to segment dendrite and special processing techniques are needed.

\subsection{Preprocessing}

The intensity profile in neuronal regions of image exhibits noise, uneven illumination and sharp variations in intensity. So preprocessing is necessary to relieve these issues.

Shot noise can be removed with a 3D median filter with a kernel size of 3 by 3 by 3 . Since the median is not sensitive to outliers, it works well to remove shot noise with little blurring. To correct uneven illumination degradation and enhance the image, we 
adopt a top-hat filter. Let $\circ$ denotes the morphological open operator; the top-hat transformation is given by

$$
h=f-(f \circ b)
$$

where $f$ is the input image and $b$ is a ball kernel. With top-hat filter, the uneven illumination is alleviated greatly. Fig. 1 illustrates the preprocessing result.

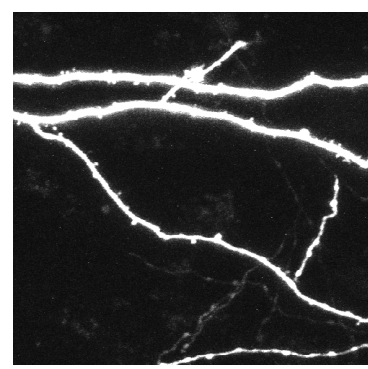

(a)

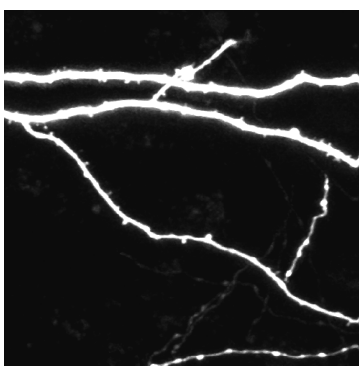

(b)

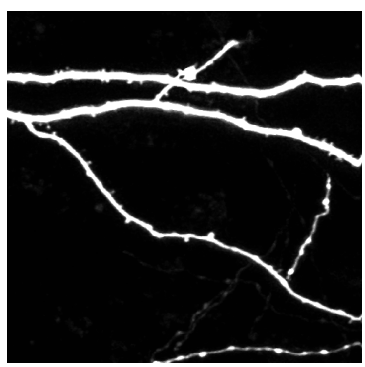

(c)

Fig. 1. Preprocessing result. (a) Original image; (b) median filter result of (a); (c) top-hat filtering result of (b). The kernel $b$ is a ball one with radius of 10 and height of 5 .

Besides, our dendrite images are characterized with sharp intensity variation which may cause some weak signals to be missed with our later level set segmentation method. To overcome this problem, a non-linear transformation is adopted

$$
f= \begin{cases}f & \text { if } f<T \\ T & \text { if } f \geq T\end{cases}
$$

where $T$ is constant of large value.

\subsection{Level Set Segmentation}

Level set is a versatile tool for analyzing and computing the motion of an active contour, and has been applied successfully in image segmentation. Generally, active contour models can be classified into two categories: edge-based models and regionbased models, both of which possess their own pros and cons [8].

Our dendrite image suffers from blurring and intensity in-homogeneity. Simple thresholding methods cannot yield satisfactory results. In this paper, we present a new level set approach based on Laplacian of Gaussian (LoG) image. Our method can effectively tackle the problems mentioned above. It is formulated as follows.

Let $C$ denote a closed contour and $\Omega$ be image domain. In level set methods $C \subset \Omega$ is represented by the zero level set of a Lipschitz function $\phi: \Omega \rightarrow \Re$, such that $\phi$ is negative for point inside $C$, positive outside $C$ and zero at $C$.

Define $\phi$ as a signed distance function, satisfying $|\nabla \phi|=1$. In an ideal case, assuming the intensity of object in an image is greater than that of the background, the 
object boundary will lie on local maxima of intensity gradient. Therefore, the negative integration of image intensity gradient along a closed curve in the image domain should achieve minimum when the curve locates in the image with local maximum gradient value. Such a curve is desired to be a zero level set curve, which can be guided by the following energy functional:

$$
E(\phi)=\int_{\Gamma(\phi=0)} \nabla\left(G_{\sigma_{1}} * I\right) \cdot \vec{n} d \Gamma
$$

where $G_{\sigma_{1}}$ is the Gaussian kernel with standard deviation $\sigma_{1}$ and $\vec{n}$ denotes the outer normal direction of the zero level set. In the formulation of $E(\phi), I$ is convolved with a Gaussian kernel for two considerations. First of all, it is more robust in the presence of noise. Second, the capture range of the active contour is extended, which can be seen from the later equation (8).

In our processing, the image domain is 3-D. To regularize the zero level set contour of $\phi$, we also need the area of the zero level set surface of $\phi$ and the weighted volume inside the zeros level set surface, which are given respectively by

$$
L(\phi)=\iiint_{\Omega} \delta(\phi)|\nabla \phi| d x d y d z
$$

and

$$
V(\phi)=\iiint_{\Omega} g(\nabla I) H(-\phi) d x d y d z
$$

where $\delta$ is the Dirac function, $H$ is the Heaviside function and $g$ is a positive nonincreasing function defined as: $g(\nabla I)=\frac{1}{1+\left|\nabla\left(G_{\sigma_{2}} * I\right)\right|^{p}}$, in which $G_{\sigma_{2}}$ is the Gaussian kernel with standard deviation $\sigma_{2}$.

Now we define the following entire energy functional

$$
F(\phi)=\lambda E(\phi)+\alpha L(\phi)+v V(\phi)
$$

By minimizing $F(\phi)$ with respect to $\phi$, we deduce the associated Euler-Lagrange equation for $\phi$. Through calculus of variations, the Gateaux derivative of the functional $F(\phi)$ can be written as

$$
\frac{\partial F}{\partial \phi}=-\delta(\phi)\left[\lambda \Delta\left(G_{\sigma} * I\right)+\alpha \nabla\left(\frac{\nabla \phi}{|\nabla \phi|}\right)+v g(\nabla I)\right]
$$

With steepest descent algorithm, we obtain the evolution equation of $\phi$ :

$$
\frac{\partial \phi}{\partial t}=\delta(\phi)\left[\lambda \Delta\left(G_{\sigma} * I\right)+\alpha \nabla\left(\frac{\nabla \phi}{|\nabla \phi|}\right)+v g(\nabla I)\right]
$$

where $\lambda, \alpha$ and $v$ are constant for weighting the different evolution force.

On the right of equation (8), the first term indicates Laplacian of Gaussian (LoG) image. LoG acts like a doublet, which aligns the curve to be closer to the zero crossings along the edge. It is intuitive that when the evolution front is around the 
zero crossings of LoG image, it will be pulled toward the zero crossings whether it locates either side of the zero crossings, until it stops on the zero crossings. The second term indicates the curvature evolution the level set, with which the surface of the zero level set stays smooth all along the evolution. The third term is a balloon force, which acts to speed up the evolution in those regions where image gradient is low or slow down the evolution when where there is high gradient. When the evolution front for zero level set is far from the true object boundary, the LoG term is small and the balloon force should dominate the zero level set evolution. Thanks to the LoG term, our model is especially effective for segmentation of blurred image.

The initial contour of level set evolution is essential to obtain an accurate final segmentation result. We initialize the active contour according to the image intensity level. That is, all voxels with intensity lower than a small constant th are initialized as background while the remains are initialized as foreground. Then a signed distance function is computed according to the initial contour for the level set evolution.

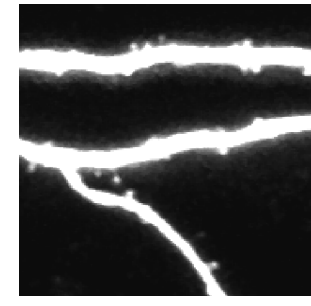

(a)

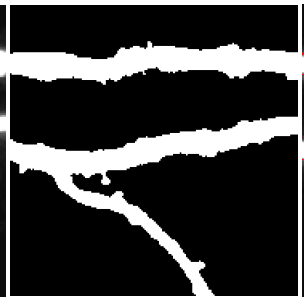

(b)

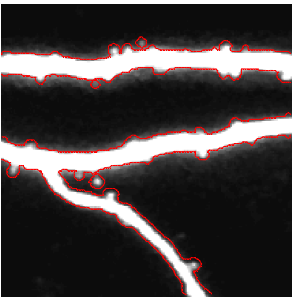

(c)

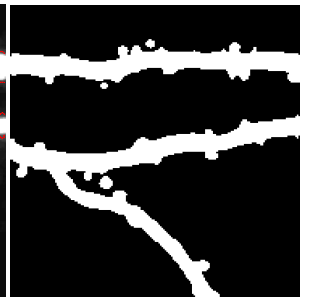

(d)

Fig. 2. Comparison of segmentation results. (a) Original image; (b) segmentation with Otsu's thresholding method; (c) level set segmentation result, the red contour indicate the boundary of the projection result of segmented object; (d) the segmentation result of (c). The images are processed in 3D and shown through maximum intensity projection (MIP).

Fig. 2 illustrates the segmentation result of a 3D dendrite patch with Otsu's method and our level set approach. It can be observed that our method can preserve the weak details of dendrite much better. The weak spine signals ignored by Otsu's method are segmented out with our method.

\section{Neuron Reconstruction and Spine Identification}

After the image processing stage, we represent the dendrite and spines by a surface model, with which the meaningful morphological measurements can be performed. The surface of the segmented dendrite is obtained by iso-surfacing. Generally, in 3D reconstruction, surface simplifying is applied to decimate the redundant mesh and reduce the tessellation density. However, in the application of our neuron dendrite visualization, considering the resolution of the neuron structure is very low and redundant surface meshes are few, surface simplifying is not adopted.

To represent the dendrite structure and identify spines, the skeleton of dendrite is extracted through a labeled three-dimensional thinning strategy. Dendritic spines, 
including detached and attached ones, are detected first. Before spine detection, the unrelated structures and short dendrite pieces emerging in the image domain are removed. Then by referring to the tip point to each detected spine, a label-based thinning strategy is presented. The erroneous branches can be removed while braches of interest are preserved. Consequently, accurate skeleton of dendrite can be obtained.

\subsection{Dendritic Spine Detection}

Detached spines are processed first. It is the photo-bleaching effects in the image acquisition process that cause the neck of some spines to be of weak intensity. Consequently, some spines are segmented to be detached from the dendrite. The detached spine can be determined according to the distance between the spine center and the dendrite backbone. If the distance is under a predefined longest spine length and the spine volume is under a maximum threshold, the corresponding spine is accepted as a true detached spine.

The detection of attached spine can be achieved by means of medial axis. Since spines are small protrusions on the dendrite, we can first determine the tips of all candidate spines according to the local extreme furthest distance from the backbone of dendrite. The medial axis can be obtained through 3D thinning [9]. Then the backbone of dendrite is extracted by removing the branches along the medial axis and smooth processing. After that, all points that are locally furthest from the backbone is determined as candidate spine tips. Finally, with the boundary locating method [10], the attached spines can be exactly segmented out.

\subsection{Dendrite Skeleton Extraction}

Dendrite skeleton consists of two parts: dendrite backbone and branch for spine. The medial axis obtained through simple $3 \mathrm{D}$ thinning is not appropriate to be taken as skeleton due to erroneous branches. Since all the spines on the dendrite are detected, this can be used as prior information to guide the 3D thinning to extract skeleton. To remove all erroneous branches and preserve branches of interest, we take the tip point to each spine as label and make use of the 3D thinning algorithm [9] to extract the dendrite skeleton.

The dendrite skeleton is extracted as follows. Firstly, the tip points for all detected attached spine are determined as labels. Secondly, we apply the iterative 3D thinning algorithm [9] to the dendrite but keep the tip point for each spine in each thinning iteration. Therefore, for each spine there would be a unique branch in the medial axis corresponding to it. Thirdly, any branch correspond to no spine is removed. Fourthly, the branch for each detached spine is determined. This is achieved by linking the center of each detached spine to the nearest point in the backbone of dendrite. After that, a post smoothing modification for skeleton may be optional.

\section{Experimental Results}

We validate our method with a set of 20 image stacks of real 3D neuron dendrite. The parameters are selected as $\Delta t=0.1, \lambda=2, \alpha=0.2, v=10, \sigma_{1}=\sigma_{2}=2, T=255$. Generally, the parameters depend on the features of the neurons being analyzed and can 
be kept the same for dataset acquired under similar conditions. As for segmentation, the performance is compared with Otsu's thresholding method. Fig. 3 illustrates the segmentation results for comparison. It can be seen that our method effectively tackles the problem of segmentation under blurring and intensity in-homogeneity. Weak blurred signals are preserved satisfactorily.

After segmentation, the 3D dendrite is reconstructed with surface representation. Then spine identification and 3D dendrite skeleton extraction are performed. Comparison with available ground-truth is performed. Fig. 4 illustrates the course of skeleton extraction. The skeleton and surface rendering of two dendrite images are shown in Fig. 5. In the skeleton, each branch denotes a detected spine and the structural information of dendrite and spine is compactly encoded. Any morphological features of interest can be conveniently extracted. This is of great significance for biological study.

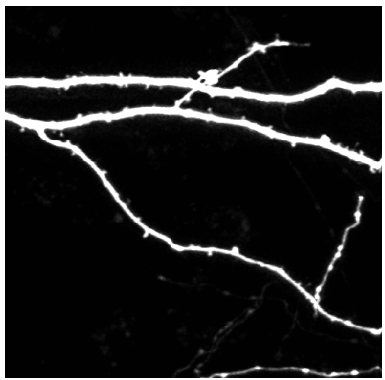

(a)

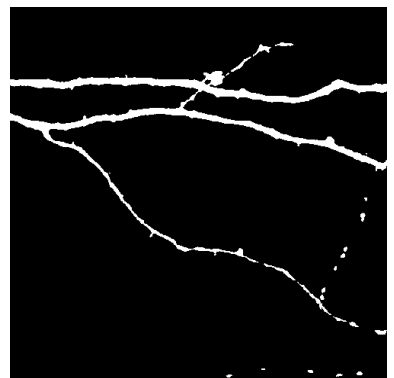

(b)

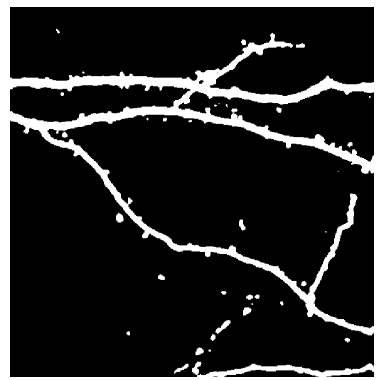

(c)

Fig. 3. Comparison between thresholding method and our level set segmentation method: (a) original image after preprocessing; (b) segmentation with Otsu's thresholding method; (c) level set segmentation result. (a), (b), (c) are shown through maximum intensity projection (MIP).

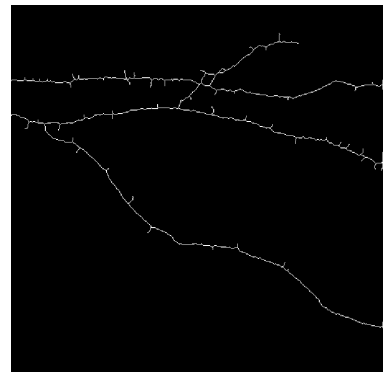

(a)

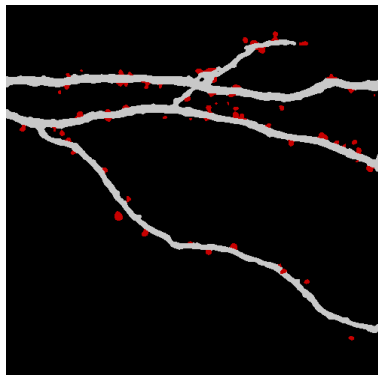

(b)

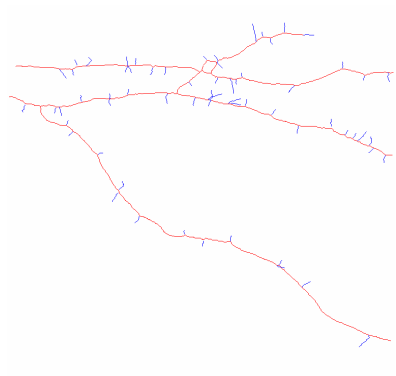

(c)

Fig. 4. 3D dendritic spine detection: (a) medial axis obtained through $3 \mathrm{D}$ thinning algorithm; (b) spine detection results, the spines highlighted in red; (c) dendrite skeleton: the backbone (red) and spines (blue). Images (a) and (b) are show through maximum intensity projection (MIP). 


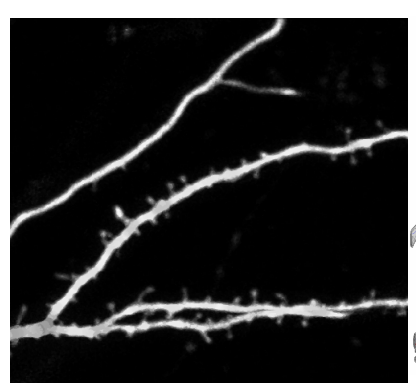

(a)

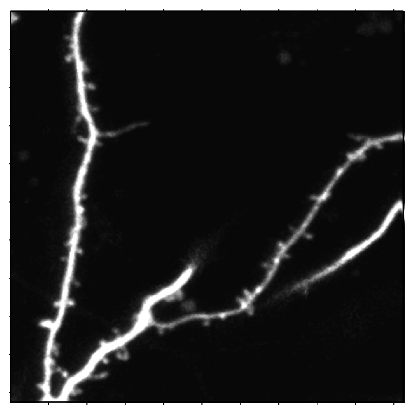

(d)

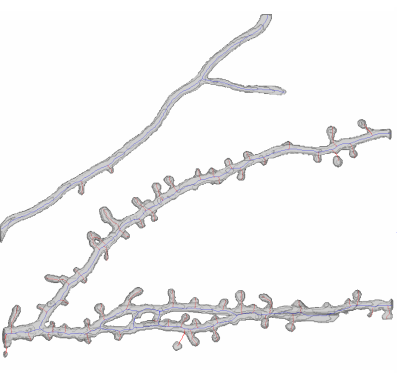

(b)

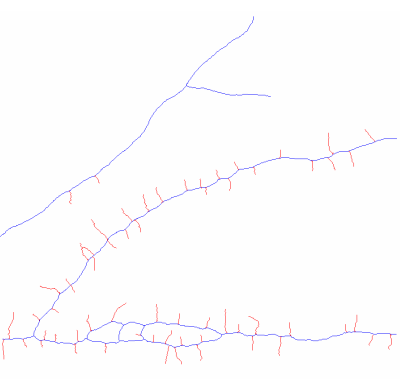

(c)

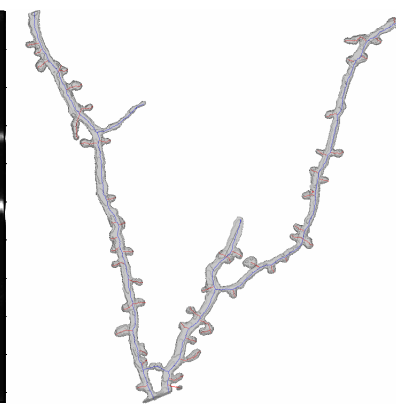

(e)

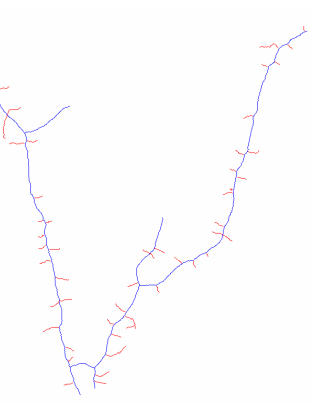

(f)

Fig. 5. Visualization of 3D neuron dendrites. (a) and (d) are two dendrite images after preprocessing shown through MIP; (b) and (e) illustrate the 3D reconstruction results of (a) and (b) respectively; (c) and (f) shows the skeletons of (b) and (e) respectively: backbone(blue) and spines (red).

\section{Conclusion}

In this paper, we describe a new automatic method for 3D dendrite reconstruction and spine identification. For segmentation of dendrite and spine components, we present a novel level set approach which can effectively deal with blurring and intensity inhomogeneity. The surface of segmented dendrite is smooth, which is very preferable for surface reconstruction. Then spines are detected and the dendrite skeleton is obtained through a label-based 3D thinning strategy to identify spines. We validate our method with true $3 \mathrm{D}$ neuron dendrite images and experimental results reveal that it works well.

\section{Acknowledgement}

The work of Houqiang Li and Wengang Zhou is supported by Program for New Century Excellent Talents in University (NCET) and open fund of MOE-Microsoft Key Laboratory of Multimedia Computing and Communication under contract No. 07122804. Dr. Zhou is partially funded by NIH R01 LM008696, R01 LM009161, 
R01 AG028928. The authors also would like to thank to Dr. Sabatini at Harvard Medical School for providing raw image data to validate the proposed system.

\section{References}

1. Nimchinsky, A., Sabatini, B.L., Svoboda, K.: Structure and function of dendritic spines. Annu. Rev. Phusiol. 64, 313-353 (2002)

2. Carlbom, I., et al.: Computer-assisted registration, segmentation, and 3D reconstruction from images of neuronal tissue sections. IEEE Trans. on Medical Imaging (1994)

3. Uehara, C., et al.: Towards automatic reconstruction of dendrite morphology from live neurons. In: IEEE Conference of the Engineering in Medicine and Biology Society (2004)

4. He, W., et al.: Automated three-dimensional tracing of hrp stained neurons from a stack of brightfield optical slices. Microscopy and Microanalysis 9, 296-310 (2003)

5. Weaver, C.M., et al.: Automated Algorithms for Multiscale Morphometry of Neuronal Dendrites. Neural Computation 16, 1353-1383 (2004)

6. Al-Kofahi, K.A., et al.: Rapid automated three-dimensional tracing of neurons from confocal image stacks. IEEE Trans. Infor. Tech. Bio. 6(2), 171-187 (2002)

7. Koh, Y.Y., Lindquist, W.B., Zito, K.: An image analysis algorithm for dendritic spines. Neural Computation 14, 1283-1310 (2002)

8. Li, C., et al.: Implicit Active Contour Driven by Local Binary Fitting Energy. In: IEEE Conf. Comput. Vis Pattern Recognit., pp. 1-7 (2007)

9. Palagyi, K., Kuba, A.: A 3D 6-subiteration thinning algorithm for extracting medial lines. Pattern Recognition letters 19 (1998)

10. Zhou, W., et al.: A new algorithm for 3D dendritic spine detection. In: International Symposium on Computational Models of Life Sciences, pp. 137-146 (2007) 